\title{
Description of a new Lathys Simon, 1884 (Araneae: Dictynidae) from Majorca, Spain
}

\author{
Описание нового вида рода Latbys Simon, 1884 \\ (Araneae: Dictynidae) с Майорки, Испания
}

\author{
Jørgen Lissner \\ Й. Аисснер \\ Natural History Museum Aarhus, Wilhelm Meyers Allé 210, Universitetsparken, 8000 Aarhus C, Denmark. E-mail: lissner@nathist.dk
}

\begin{abstract}
KEY WORDS: Aranei, spiders, stigmatisata-group, new species, description.
КЛЮЧЕВЫЕ СЛОВА: пауки, группа видов stigmatisata, новый вид, описание.
\end{abstract}

ABSTRACT. A new spider species, Lathys mallorcensis sp.n. is described from Majorca. The species seems confined to hypogean environments of high ground karst landscapes of the Tramuntana mountain range. The new species belongs to the $L$. stigmatisata group and differs from its Mediterranean sibling species by its small size, shape of terminal part of conductor and choice of habitat. The occurrence of Lathys species in the Balearic Islands is reviewed.

How to cite this article: Lissner J. 2018. Description of a new Lathys Simon, 1884 (Araneae: Dictynidae) from Majorca, Spain // Arthropoda Selecta. Vol.27. No.2. P.129-137. doi: 10.15298/arthsel. 27.2.04

РЕЗЮМЕ. Новый вид пауков, Lathys mallorcensis sp.n. описан с Майорки. Похоже, что распространение вида ограничено наземными карстовыми ландшафтами горного хребта Трамонтана. Новый вид принадлежит к группе L. stigmatisata и отличается от ближайших средиземноморских видов мелкими размерами, формой терминальной части кондуктора и типом местообитания. Дан обзор находок видов Lathys на Балеарских островах.

\section{Introduction}

Lathys Simon, 1884 is a genus of spiders in the Dictynidae family with 46 accepted species [World Spider Catalog, 2017]. Some of the characters that distinguish Lathys include eight eyes with anterior median eyes small, undivided cribellum, low clypeus about the diameter of an anterior median eye, short, not converging endites, long tarsal trichobothria, a modified cymbium, absence of tibial spines on male palp, terminal conductor screw or spine like, relatively long copulatory ducts, and relatively large spermathecae compared to most members of Dictynidae [Almquist 2006, Kaston, 1978; Marusik pers. comm., Nentwig et al., 2017; Zhang et al., 2012]. The Lathys stigmatisatagroup was first delineated by Lehtinen [1967] as a
Lathys puta group. Later Marusik et al. [2006] unveiled the rather peculiar and unique structures in the conformation of the male palp in this group. They found that the conductor is with two arms, one screwed and one coiled and that the tibial apophyses function as a bracket securing the screwed conductor in position. The combined distribution of the L. stigmatisata-group is Holarctic (see Marusik et al. [2006] for map). Attempts to identify Lathys specimens recently collected in Majorca failed and the specimens were recognized as belonging to a new species of the L. stigmatisata-group. During the identification process 14 Palaearctic species and 2 Nearctic species were categorized as belonging to the group using data available in the literature. However, the exact number of species in the group is unknown since some species (L. balestrerii Caporiacco, 1934, L. arabs Simon, 1910) may have been erroneously synonymised with $L$. stigmatisata (Menge, 1869) [Bosmans et al., 2009; Kovblyuk et al., 2014]. One member of the group, L. jubata (Denis, 1947), is possibly a synonym of L. stigmatisata [Ledoux et al., 2008]. New species have been added to the group as late as 2012 (L. subalberta Zhang, Hu et Zhang, 2012) [Zhang et al., 2012] and 2014 (L. lehtineni Kovblyuk, Kastrygina et Omelko, 2014) [Kovblyuk et al., 2014]. There are still many undescribed species [Marusik et al., 2009; Koçyi it et al., 2016] or insufficiently described species such as $L$. truncata Danilov, 1994 and L. maculosa (Karsch, 1879).

The aim of this study is to describe the new Majorcan Lathys species. Specimens were found primarily in hypogean environments: in caves, deep in scree and among layered stones deeper in the ground. All records are from karst landscapes of the Tramuntana mountain range. A further aim is to review the occurrences of Lathys species in the Balearic Islands.

\section{Materials and methods}

Specimens were collected by hand by turning stones in caves and examining surfaces of layered rocks in the ground. 


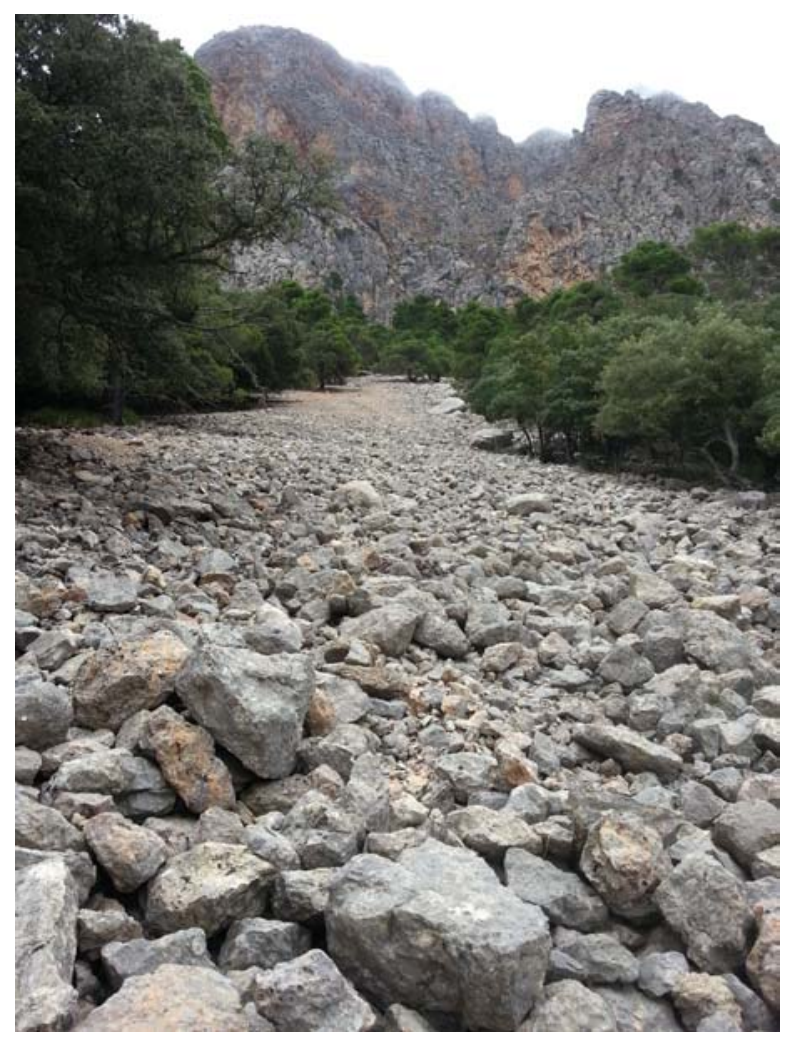

Fig. 1. Steep scree slope at Puig Major, the locality at which site the type specimens were collected.

Рис. 1. Крутой склон с осыпью в Puig Major, местонахождении, где были собраны типовые экземпляры.

In scree specimens were collected from the surfaces of stones lifted away one by one down to a depth of one meter (Fig. 1). Illustrations were created from photos of selected features using a Leica Wild M10 stereomicroscope fitted with Leica DFC425 digital camera connected to a computer with Leica Application Suite software v. 4.3.0, Zerene Stacker software v. 1.04 and the vector graphics editor Inkscape v. 0.92 .

The terminology and abbreviations of palp and epigyne morphology follow Kovblyuk et al. [2014] and is based on Marusik et al. [2006, 2009], also used by Zhang et al. [2012]: Co - copulatory opening; $\mathrm{Ct}$ - terminal part of conductor; $\mathrm{Ctt}$ - tip of $\mathrm{Ct}$; $\mathrm{Dt}$ - dorsal tibial apophysis; $\mathrm{Eb}$ - Embolar base; Em - Embolus; Fd - fertilization duct; Ia - apical portion of insemination duct; Ib - basal portion of insemination duct; $\mathrm{Ra}$ - intermediate (dorsolateral) apophysis; $\mathrm{Sp}$ - spermathecae; $\mathrm{Va}$ - ventral apophysis. Further abbreviations used: CL — carapace length; CW — carapace width; OL — opisthosoma length; TL total length; AME - anterior median eyes; ALE - anterior lateral eyes; PME - posterior median eyes; PLE - posterior lateral eyes; MSS - mesovoid shallow substratum; CJL - coll. Jørgen Lissner; NHMD - Natural History Museum of Denmark; Sd — seminal duct; Ss - origin of seminal duct; UTM - Universal Transverse Mercator coordinate system, Majorca is situated in UTM zone 31 in latitude band $\mathrm{S}$, precision is given to $10 \mathrm{~m}$ which is approximately the precision of the Garmin Oregon 450 GPS used to locate positions.

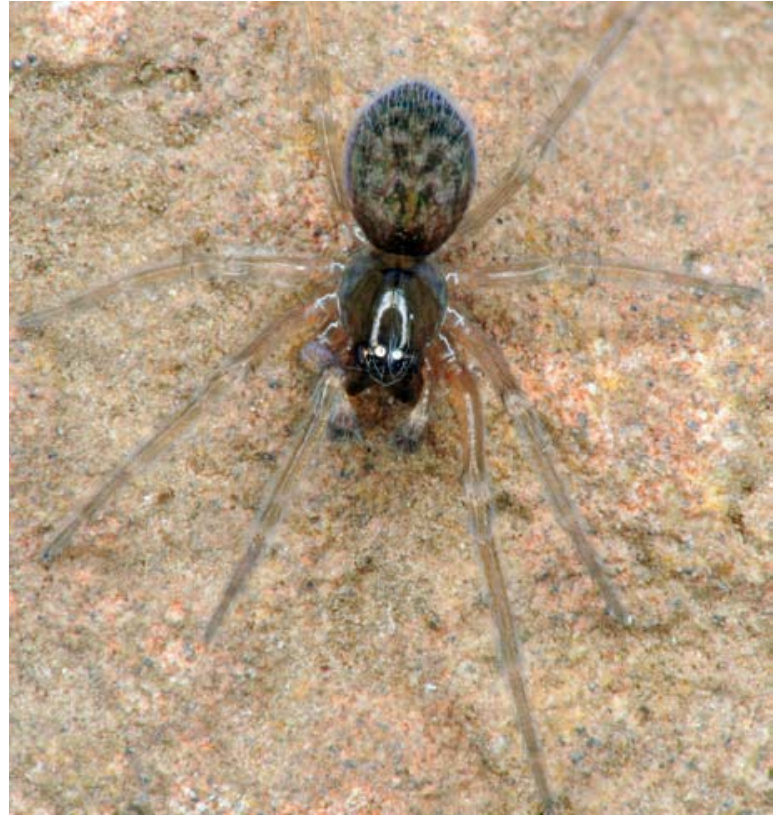

Fig. 2. Lathys mallorcensis sp.n., habitus of live male.

Рис. 2. Lathys mallorcensis sp.n., внешний вид живого самца.

\section{Taxonomy}

Lathys mallorcensis sp.n.

Figs 2-11.

TYPE MATERIAL: SPAIN. Majorca, Holotype $\sigma^{7}$, Puig Major, scree at Es Cards Colers (Fig. 1), $890 \mathrm{~m}$ (UTM 31S 48225 440469), 29.X.2014 (specimen matured in captivity around 1.III.2015), J. Lissner leg. Paratypes $2 \sigma^{\top} \sigma^{\top}$ (matured in captivity), 3 우 same locality as holotype, 9.X.2015, J. Lissner leg, CJL11039; $1 \sigma^{7}$ : Majorca, Pollença, Cova des Boc, cave, 490 m (UTM 31S 49869 441048), 6.IV.2016, J. Lissner leg, CJL-11400; 2 ○ $\sigma^{7}$ : Majorca, under layered stones along track west of the Cuber reservoir towards Coll de l'Ofre, 750 m (UTM 31S 48012 440224), 29.X.2014 (both specimen matured in captivity c. 20.IV.2016), J. Lissner leg, CJL-11491; 1 O: Majorca, Pollença, Cova Morella, cave, $505 \mathrm{~m}$ (UTM 31S 49863 441048), 6.IV.2016 (specimen matured in captivity around 1.V.2016), J. Lissner leg, CJL-11500; $1 \sigma^{7}$ : Majorca, Massanella, Coll des Prat, rock steppe, cavity in ground under large stone, $1210 \mathrm{~m}$ (UTM 31S 48733 440649), 26.IV.2017, J. Lissner leg, CJL-11781.

The holotype and paratypes are deposited at NHMD.

Additional material examined: Lathys sp. aff. narbonensis (Simon, 1876), $1 \sigma^{7} 3$ 우, Majorca, Sant Elm, open pine forest with understory bushes and weeds, $30 \mathrm{~m}$, (UTM $31 \mathrm{~S} 44467$ 438097), 19.IV.2013, J. Lissner leg, CJL-9954. Lathys teideensis Wunderlich, 1992, $2 \sigma^{7} \sigma^{7}$, Tenerife, Las Raices, pine forest, $1100 \mathrm{~m}$ (UTM 28R 36511 314508), 3.IX.2015, J. Lissner leg, CJL-10927.

DIAGNOSIS. The new species belongs to the $L$. stigmatisata-group. Males differ from its nearest sibling species (L. lehtineni, L. arabs, L. stigmatisata) by the long, straight tip of terminal part of screwed conductor which is parallel with tibia in retrolateral view (perpendicular in sibling species). Females differ from these species and L. subviridis Denis, 1937 by small body size and with the exception of $L$. arabs also by larger distance between epigynal openings. The species also differs from related species by occupying 


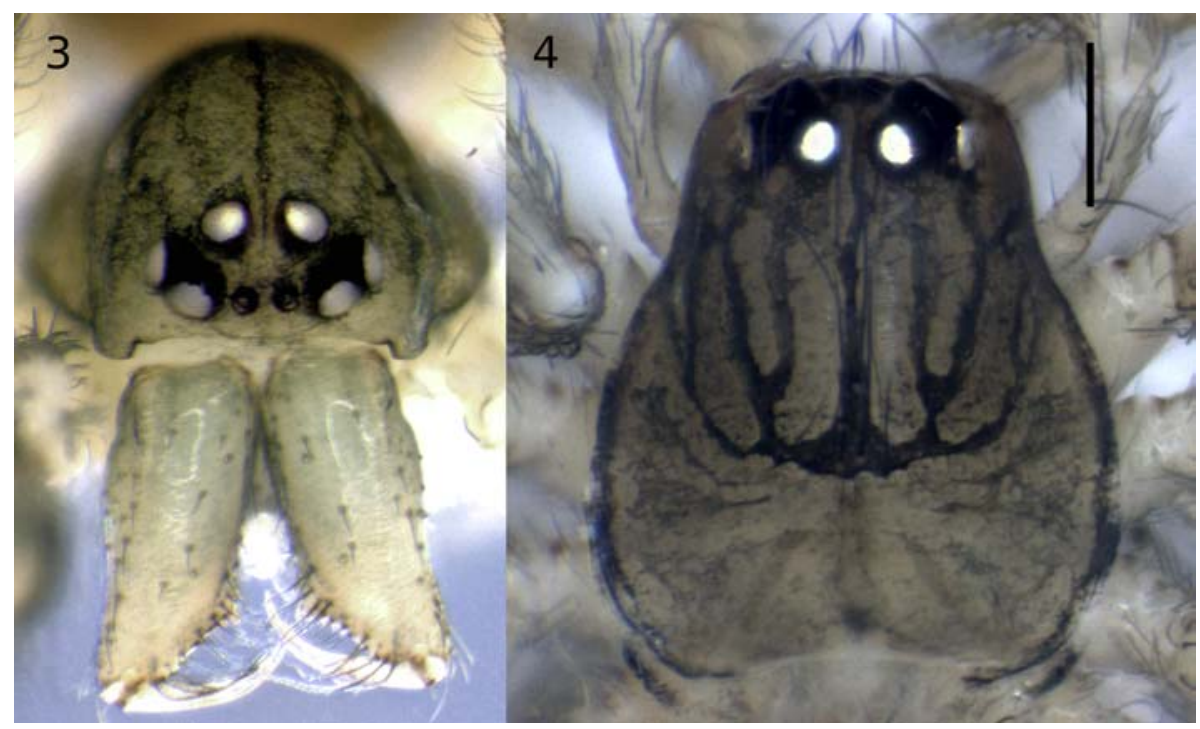

Fig. 3-4. Lathys mallorcensis sp.n.: 3 - male facies; 4 - female prosoma. Scale $0.2 \mathrm{~mm}$.

Рис. 3-4. Lathys mallorcensis sp.n.: 3 - лицевая сторона самца; 4 - головогрудь самки. Масштаб 0,2 мм.

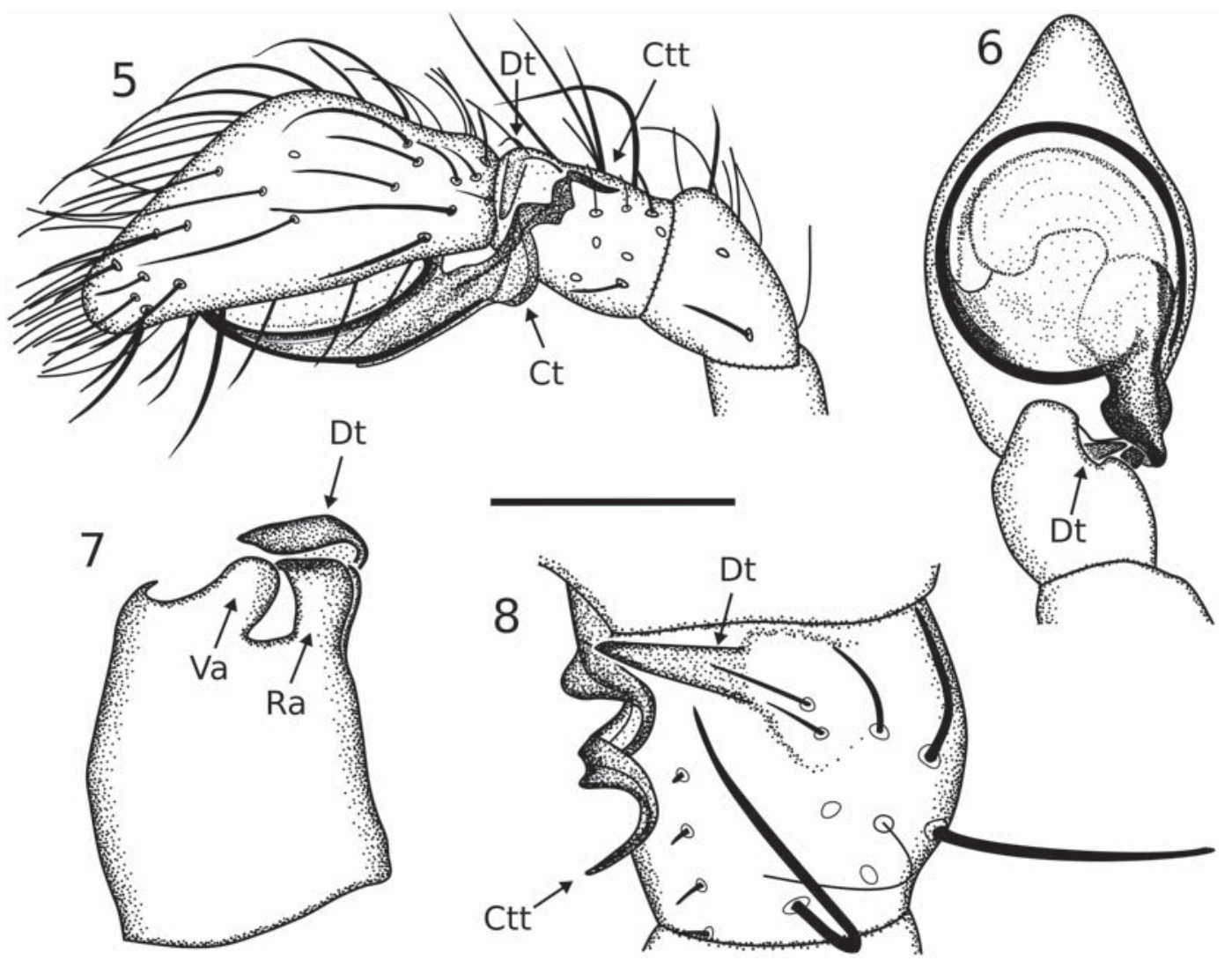

Fig. 5-8. Lathys mallorcensis sp.n.: 5 - male palp, retrolateral view; 6 - same, ventral view, hairs omitted; 7 - tibial apophyses; 8 dorsal aspect of palpal tibia. Scale $5-7-0.2 \mathrm{~mm}, 8-0.1 \mathrm{~mm}$.

Рис. 5-8. Lathys mallorcensis sp.n.: 5 - пальпа самца, ретролатерально; 6 - то же, вентрально, без волосков; 7 - вырост голени пальпы; 8 - голень пальпы, дорсально. Масштаб: 5-7 - 0,2 мм, $8-0,1$ мм. 


\begin{tabular}{|c|c|c|c|c|c|c|c|c|c|c|c|c|c|}
\hline 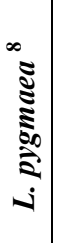 & 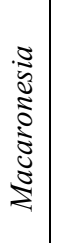 & $\stackrel{0}{0}$ & 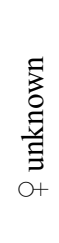 & $\stackrel{n}{=}$ & $\begin{array}{c}\tilde{E} \\
0 \\
\vdots \\
\Xi \\
\Xi \\
\\
0+\end{array}$ & 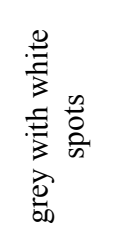 & $\begin{array}{c}\xi \\
\vdots \\
\vdots \\
\Xi \\
\Xi \\
0+\end{array}$ & 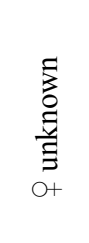 & $\begin{array}{c}\text { है } \\
0 \\
\text { ह } \\
\text { 吾 } \\
\text { O+ }\end{array}$ & 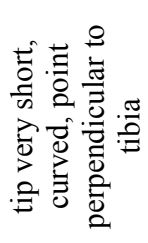 & $\begin{array}{l}\overrightarrow{0} \\
\text { 岕 } \\
\text { : }\end{array}$ & 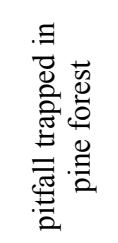 & 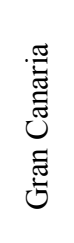 \\
\hline 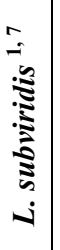 & $\stackrel{\widetilde{Z}}{*}$ & 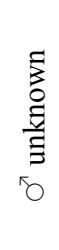 & r. & 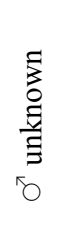 & $\stackrel{m}{i}$ & 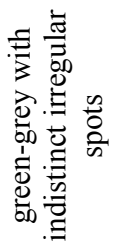 & 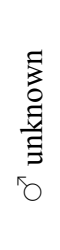 & 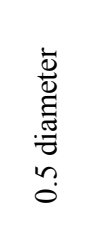 & a. & 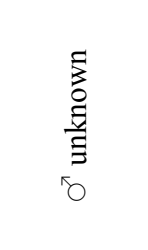 & 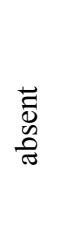 & 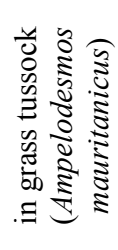 & $\begin{array}{l}\frac{\pi}{\tilde{D}_{0}} \\
\frac{.00}{<}\end{array}$ \\
\hline 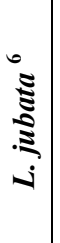 & 号 & 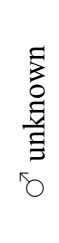 & $\exists$ & 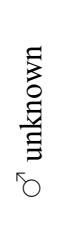 & $\stackrel{\leftrightarrow}{i}$ & 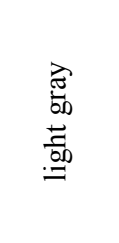 & 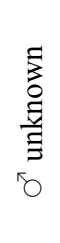 & 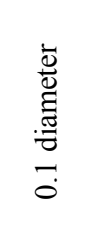 & - & $\begin{array}{l}\text { है } \\
\text { O } \\
\text { 章 } \\
{ }^{\circ}\end{array}$ & 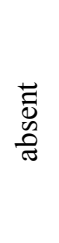 & 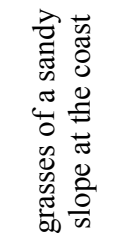 & 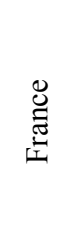 \\
\hline $\mid$ & 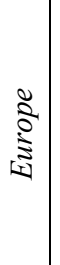 & $\stackrel{\circ}{-}$ & 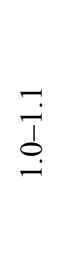 & $N$ & $\begin{array}{l}\stackrel{n}{i} \\
i \\
i n \\
i\end{array}$ & 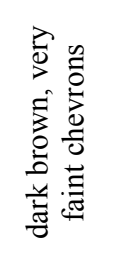 & 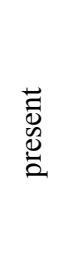 & 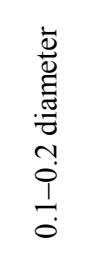 & - &  & 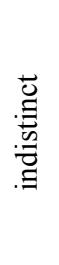 & 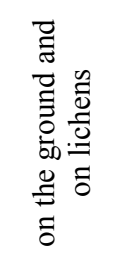 & $\begin{array}{l}\text { О. } \\
\text { : } \\
\text { : }\end{array}$ \\
\hline 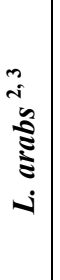 & 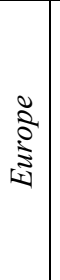 & a. & a. & $\begin{array}{l}\tilde{n} \\
\stackrel{i}{d}\end{array}$ & $\begin{array}{l}\hat{n} \\
i \\
i\end{array}$ & 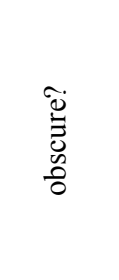 & a. & 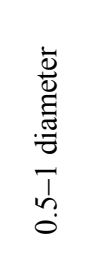 & $N$ & 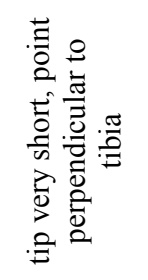 & $\begin{array}{l}\frac{\pi}{\tilde{J}} \\
\stackrel{\Xi}{\Xi} \\
\end{array}$ & 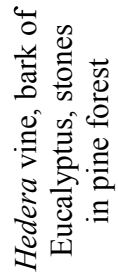 & 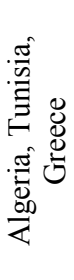 \\
\hline 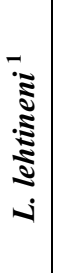 & 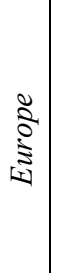 & $\stackrel{\circ}{-}$ & $\begin{array}{l}\vec{T} \\
\bar{d} \\
a \\
0\end{array}$ & $\stackrel{\sim}{\mathrm{N}}$ & 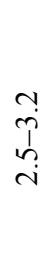 & 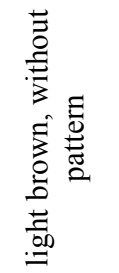 & $\begin{array}{l}\overrightarrow{\tilde{J}} \\
\text { ते }\end{array}$ & 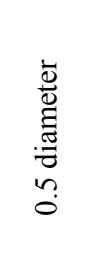 & - & 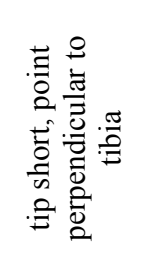 & 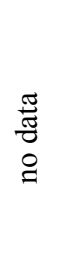 & 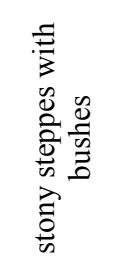 & 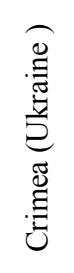 \\
\hline 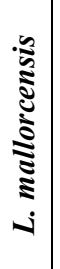 & 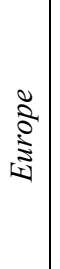 & $\begin{array}{l}\exists \\
\bar{d} \\
\sigma \\
\delta\end{array}$ & $\frac{a}{0}$ & $\begin{array}{l}\stackrel{0}{ } \\
\stackrel{i}{1} \\
\stackrel{i}{i}\end{array}$ & 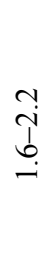 & 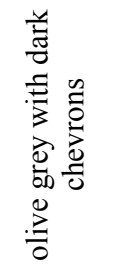 & 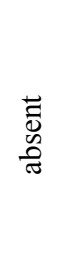 & 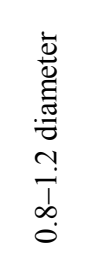 & - & 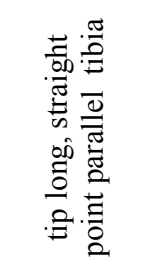 & 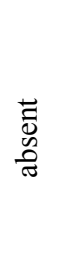 & 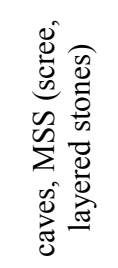 & 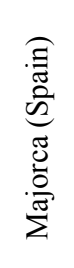 \\
\hline 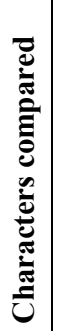 & 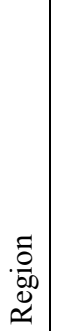 & $\begin{array}{l}{ }^{\circ} \mathrm{O} \\
\overrightarrow{\mathrm{U}}\end{array}$ & $\begin{array}{l}{ }^{\circ+} \\
\mathrm{U}\end{array}$ & $\begin{array}{l}{ }^{5} \mathrm{O} \\
\mathrm{H}\end{array}$ & $\stackrel{\text { O+ }}{\vec{H}}$ & 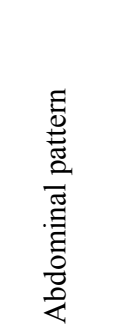 & 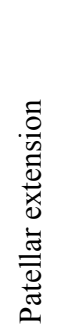 & 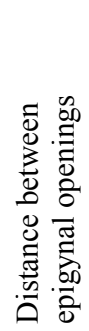 & 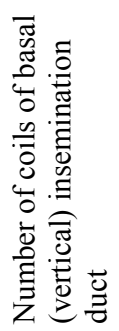 & 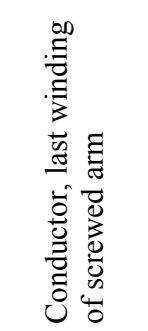 & 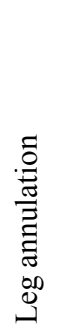 & 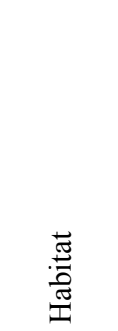 & 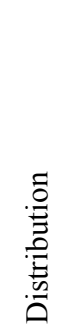 \\
\hline
\end{tabular}




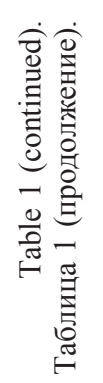

\begin{tabular}{|c|c|c|c|c|c|c|c|c|c|c|c|c|c|}
\hline 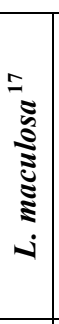 & 离 & $\begin{array}{l}\frac{\pi}{\pi} \\
\frac{\pi}{\sigma} \\
\varrho\end{array}$ & $\begin{array}{l}\frac{\pi}{\pi} \\
\frac{\pi}{\delta} \\
\stackrel{\Xi}{\Xi}\end{array}$ & $\begin{array}{l}n \\
i \\
1 \\
\stackrel{n}{i} \\
i\end{array}$ & $\begin{array}{l}n \\
i \\
1 \\
o \\
i\end{array}$ & 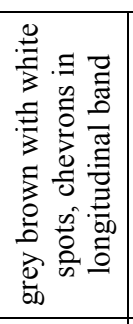 & $\begin{array}{l}\overrightarrow{\tilde{\Xi}} \\
\text { है } \\
\vec{\sigma}\end{array}$ & 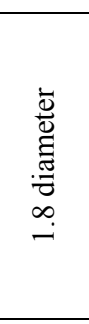 & $N$ & 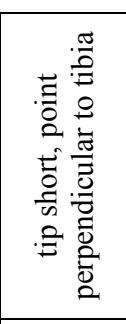 & 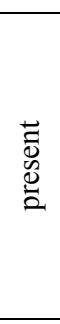 & 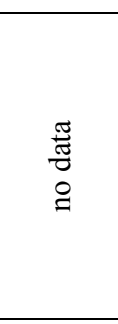 & 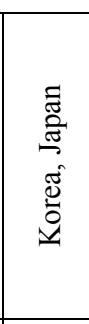 \\
\hline 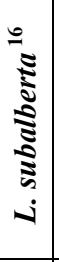 & चू & तે & $\begin{array}{l}0 \\
\infty \\
0\end{array}$ & $\stackrel{\infty}{i}$ & $\begin{array}{l}\text { N } \\
\text { I }\end{array}$ & 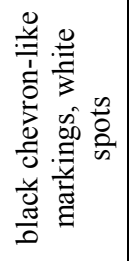 & 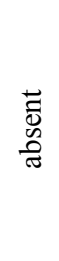 & 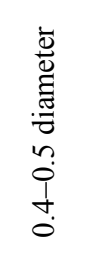 & $N$ & 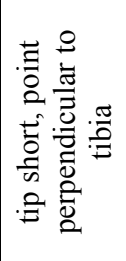 & 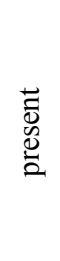 & 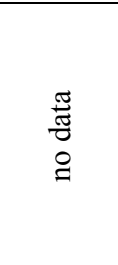 & 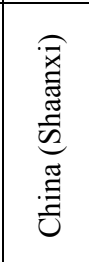 \\
\hline 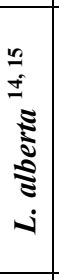 & 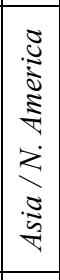 & $\because$ & $\because$ & $\stackrel{\sim}{r}$ & $\begin{array}{l}0 \\
\dot{n} \\
n \\
i\end{array}$ & 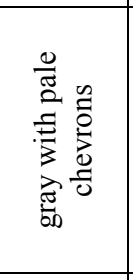 & $\begin{array}{l}\overrightarrow{\vec{J}} \\
\text { ते } \\
\text { त् }\end{array}$ & 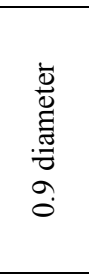 & $N$ & 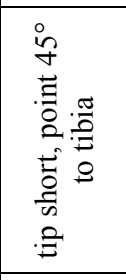 & 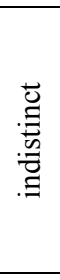 & 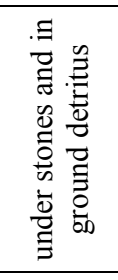 & 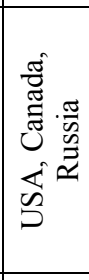 \\
\hline 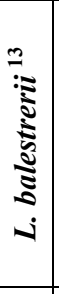 & $\frac{\pi}{4}$ & $\stackrel{n}{\rightarrow}$ & $\stackrel{9}{-}$ & $\tilde{i}$ & $\stackrel{n}{i}$ & 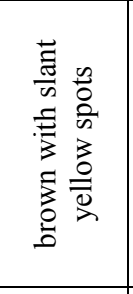 & $\begin{array}{l}\overrightarrow{\vec{J}} \\
\text { d. } \\
\text { त्र }\end{array}$ & 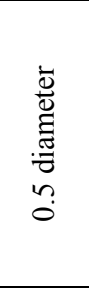 & $N$ & 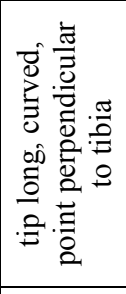 & $\begin{array}{l}\text { च्च } \\
\text { हू } \\
\text { तै }\end{array}$ & 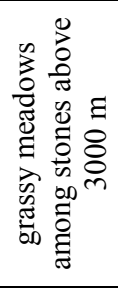 & 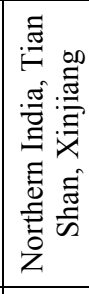 \\
\hline 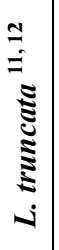 & $\frac{\Xi}{8}$ & 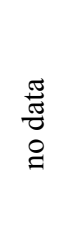 & $\stackrel{\sim}{\Im}$ & $\begin{array}{l}\frac{\pi}{\pi} \\
\frac{\pi}{\sigma} \\
\varrho\end{array}$ & 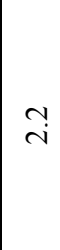 & 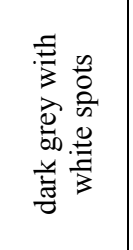 & 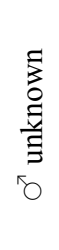 & 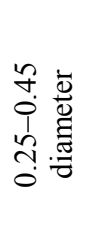 & $\vec{i}$ & 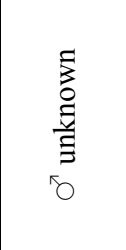 & a. & 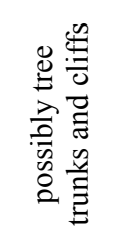 & 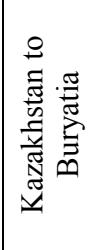 \\
\hline 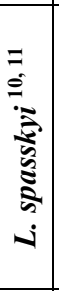 & $\frac{\pi}{4}$ & 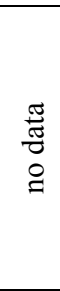 & $\stackrel{\circ}{=}$ & $\begin{array}{l}\frac{\pi}{\tilde{J}} \\
\text { @ } \\
\varrho\end{array}$ & $\stackrel{\infty}{i}$ & 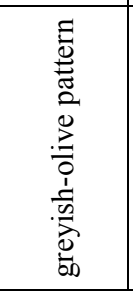 & $\begin{array}{l}\overrightarrow{\bar{D}} \\
\text { ते } \\
\text { त् }\end{array}$ & 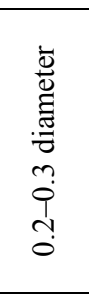 & $N$ & 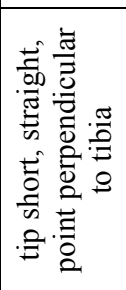 & $\begin{array}{l}\overrightarrow{\vec{\nu}} \\
\text { है } \\
\text { त् }\end{array}$ & 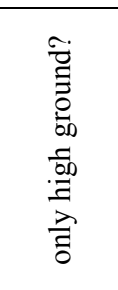 & 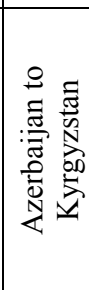 \\
\hline 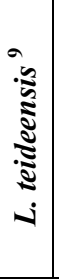 & 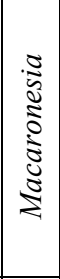 & $\stackrel{n}{r}$ & 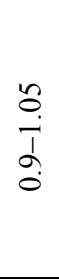 & $\stackrel{\sim}{N}$ & $\begin{array}{l}0 \\
i \\
i \\
n \\
i\end{array}$ & 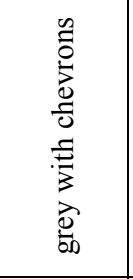 & $\begin{array}{l}\vec{\Xi} \\
\text { ते } \\
\text { ते }\end{array}$ & r. & 0 & 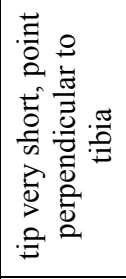 & 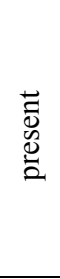 & 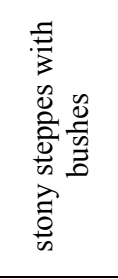 & $\begin{array}{l}\stackrel{0}{0} \\
\stackrel{0}{0} \\
\stackrel{0}{0}\end{array}$ \\
\hline 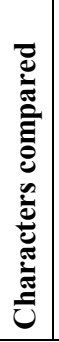 & 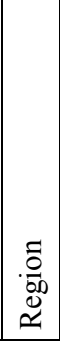 & $\begin{array}{l}0 \\
0 \\
\end{array}$ & $\begin{array}{l}\text { O+ } \\
\text { U }\end{array}$ & $\begin{array}{l}{ }^{\circ} \\
\stackrel{H}{H}\end{array}$ & $\stackrel{\mathrm{O}}{\mathrm{H}}$ & 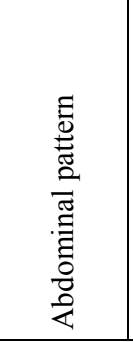 & 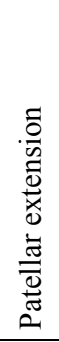 & 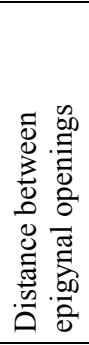 & 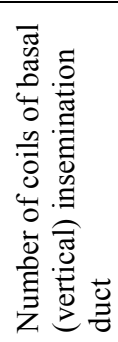 & 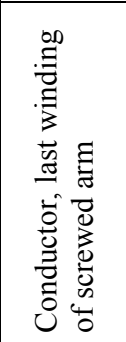 & 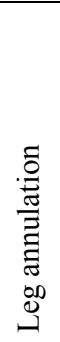 & 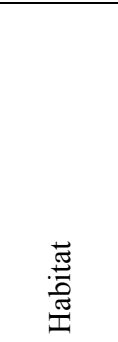 & 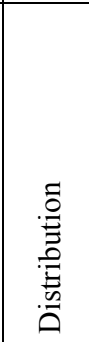 \\
\hline
\end{tabular}

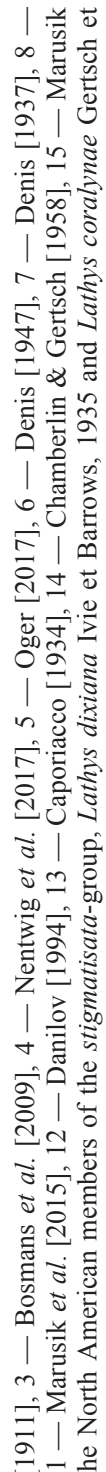

可要

홍ㅎㅎ

$\sqrt{2} \frac{1}{3}$

氙需尔

1

이

商金:

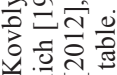
I

긍

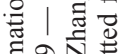

항

훙응

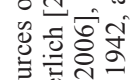


Table 2. Lathys mallorcensis sp.n. Counts of cheliceral teeth (ranging from 3-5) on promargin (PM) and retromargin (RM).

Таблица 2. Lathys mallorcensis sp.n. Число зубцов хелицер (от 3 до 5) на переднем (PM) и заднем (RM) крае.

\begin{tabular}{llllllll} 
No of teeth & 3 & 4 & 5 & & 3 & 4 & 5 \\
\hline$\sigma^{7} \mathrm{PM}$ & 1 & 5 & 4 & O PM & 1 & 4 & 3 \\
$\mathrm{O}^{7} \mathrm{RM}$ & 0 & 6 & 4 & O RM & 2 & 4 & 2 \\
\hline
\end{tabular}

Ten chelicers (five specimens) were counted for males, eight (four specimens) for females.

Table 3. Lathys mallorcensis sp.n., lengths of leg segments and carapace ( $\mathrm{mm}$ ) of adult male and female. The ratio of total leg length (coxa and trochanters excluded) to carapace length $(\mathrm{L} / \mathrm{C})$ is calculated.

Таблица 3. Lathys mallorcensis sp.n., длина члеников ног и головогруди половозрелого самца и самки. Указано также соотношение общей длины ноги (без тазика и вертлуга) к длине головогрудного щита.

\begin{tabular}{ccccccccc} 
Leg & Femur & Patella & Tibia & Metatarsus & Tarsus & Total & Carapace & L/C ratio \\
\hline $\mathrm{I}$ O $^{7}$ & 0.96 & 0.36 & 0.83 & 0.61 & 0.42 & 3.18 & 0.91 & 3.5 \\
$\mathrm{I}+$ & 0.71 & 0.31 & 0.51 & 0.45 & 0.28 & 2.26 & 0.92 & 2.5 \\
\hline
\end{tabular}

Adult male and female are taken with approximately same size of carapace; one leg of each sex is measured.

hypogean environments, all other species are epigean. Further characters that separate the new species from Palaearctic members of the stigmatisata-group are listed in Table 1.

DESCRIPTION. Male. Measurements: $(\mathrm{n}=5$, average value with range in parenthesis): TL: 2.26 (2.12.6), CL: 0.98 (0.9-1.1), CW: 0.78 (0.7-0.9), OL: 1.18 (1.1-1.3). Carapace olive green with dark grey reticulations and striae (Figs. 2-4). Chelicerae light olive green (Fig. 3). Sternum coloured as carapace with dark reticulations at margins. Dorsum of abdomen olive green, dark grey marking anteriorly followed by chevrons broken in to dark blotches along midline (Fig. 2). Legs whitish, very slightly darkened apically on tibia IV and metatarsi I-IV.

Posterior eye rows slightly procurv in dorsal view, anterior row slightly recurved, posterior row procurved as seen from in front. Eyes rather large compared to most congeners, the distance between AME is just 0.75 of the diameter (Fig. 3). PME separated by their diameter, from the PLE by 1.5 times their diameter. Eye sizes $(n=1)$ : AME 0.025, ALE 0.075, PME 0.072, PLE 0.080 . Clypeus narrow, about equal to the diameter of one AME (Fig. 3).

Chelicerae with (3)4-5 teeth of variable size on pro- and retromargin (Table 2). Number of teeth vary among individuals and between right and left chelicera. Promargin typically with 2 large teeth and 1-3 smaller teeth or denticles, while teeth of retromargin generally smaller with some represented by denticles. Males with greater differences in tooth sizes than females. Male relatively long-legged compared to female. Leg I 3.5 times the length of the carapace versus 2.5 times in the female (Table 3). Terminal part of conductor forms an open spiral with well separated, slender windings. The section at the very tip relatively long, straight and parallel with palpal tibia in retrolateral view (Fig. 5). Palpal patella without an extension.
Tibia with a distinct, well-developed dorsal apophysis having straight edges forming an elongated triangle perpendicular to axis of tibia, more than twice as long as wide when viewed in dorsal view (Fig. 8). In retrolateral view it is seen to point towards terminal, screwed conductor (Fig. 4). The apex of the dorsal apophysis is also visible in ventral view (Fig. 6). Ventral and retrolateral tibial apophyses obscured by the terminal conductor and difficult to observe unless the bulb with conductor is removed as done to illustrate all tibial apophyses in Fig. 7. A longitudinal furrow of the ventral apophysis (sensu Marusik [2006]) is indistinct and is only vaguely discernible using a microscope (furrow not drawn in Fig. 7, based on stereomicroscope photo. Base of embolus in 7 o'clock position when viewing

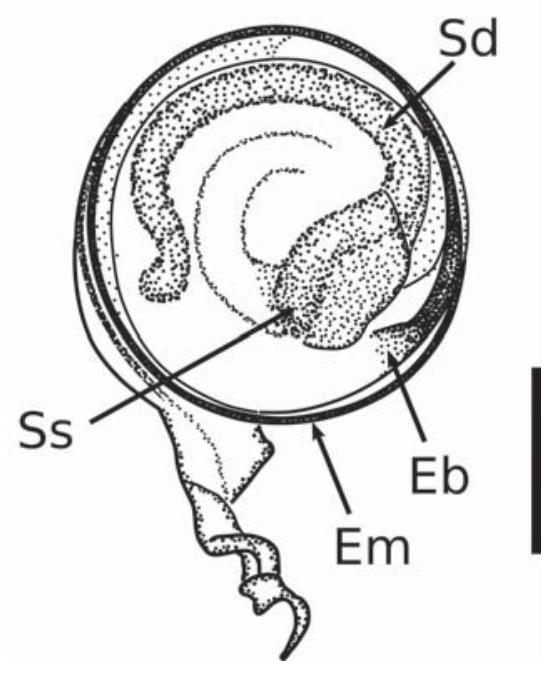

Fig. 9. Lathys mallorcensis sp.n., dorsal aspect of bulbus. Рис. 9. Lathys mallorcensis sp.n., бульбус дорсально. 

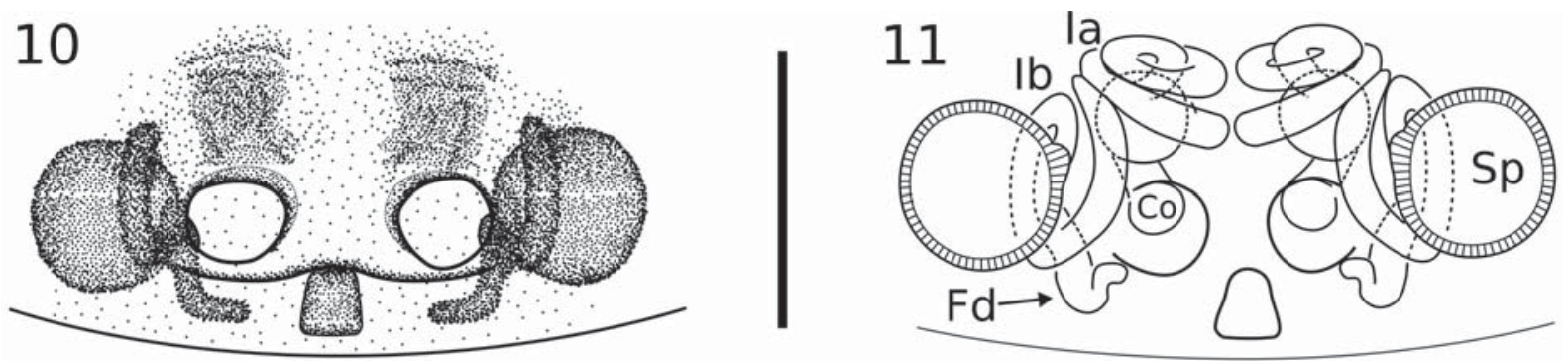

Fig. 10-11. Lathys mallorcensis sp.n.: 10 - epigyne; 11 - vulva. Scale $0.1 \mathrm{~mm}$.

Рис. 10-11. Lathys mallorcensis sp.n.: 10 - эпигина; 11 - вульва. Масштаб 0,1 мм.

detached bulb dorsally (Fig. 9). Seminal duct relatively wide and of uniform thickness, lacking a seminal loop.

Female. Measurements: $(n=4$, average value with range in parenthesis): TL: 1.85 (1.6-2.2), CL: 0.79 (0.7-0.9), CW: 0.64 (0.6-0.7), OL: 1.13 (0.9-1.4). Only larger females overlap in size with smaller males. Colouration as in male except for carapace markings in front of fovea forming longitudinal cells, quite distinct in some specimens (Fig. 4). Width of clypeus as in males. Eye sizes $(n=1)$ : AME 0.024, ALE 0.067, PME 0.069 , PLE 0.071 . Chelicerae with $3-5$ teeth on both margins with 4 being most frequent in both rows (Table 2). The teeth nearest fang socket are mostly contiguous the most distal sometimes free. The second promarginal tooth distal to fang socket much larger than the remaining, more or less similar-sized teeth. Some teeth may be represented by denticles only. Legs whitish, not annulated or darkened apically on segments. Epigyne as in Fig. 10. The epigynal openings approximately circular and separated by $0.8-1.2$ diameter. With a small sclerotized central plate near epigastric furrow (Fig. 10). The length/width ratio of the plate varies among individuals and is also dependent on viewing angle. The round spermathecae, inseminations ducts and fertilization ducts are partly visible through the cuticle. The copulatory openings are situated in the epigynal atrium, each copulatory opening slightly anterior to the centre of the epigynal openings (Fig. 11). Insemination ducts not funnel-shaped as diameter of copulatory opening only slightly larger than diameter of ducts. Inseminations ducts each possess a globular swelling (a gland?) about $0.01 \mathrm{~mm}$ after copulatory openings. Swellings are difficult to discern when viewing vulva in dorsal view since they are partly enclosed by the coil of duct windings. They are drawn with dotted lines in Fig. 11. Ducts continue from near top of swellings and enter spermathecae after two horizontal and one vertical winding (Fig. 11).

TYPE LOCALITY. Majorca, Puig Major at Es Cards Colers.

DISTRIBUTION: The species is only known from the Serra de Tramuntana mountain range of Majorca. Specimens have been collected between Soller and Pollença at 500-1200 m elevation (Fig. 12).

HABITAT AND ECOLOGY. The holotype specimen and paratypes were found among stones on a steep slope with scree composed of large layered stones with nearest vegetation at least $5 \mathrm{~m}$ away (Fig. 1). Specimens were scarce in top layers (only one subadult male) becoming more common deep in scree, here it co-occurred with troglophilic species such as Leptoneta infuscata Simon, 1872 and Mysmena leucoplagiata (Simon, 1879). Females were only found deep in the scree. Further records are from the underside of layered stones deeply embedded in a creek bank (subadult male), in a cavity beneath a square meter large stone on high ground (Massanella, $1200 \mathrm{~m}$ ) and from under stones on a cave floor about 5-20 m from entrance in subdued light (male and female), here also with Leptoneta infuscata. Thus it seems that the species prefers dark and relatively cold places and is not associated with vegetation. Females seem to avoid top the layers, except in caves and perhaps their generally smaller sizes compared to males is related to food being scarcer in caves and in the crevices deeper in the ground. In captivity it produces a small cribellate web and readily catches small flies (in one instance also a springtail) if prey size is smaller than the spider itself.

PHENOLOGY. $\sigma^{7}$ III-IV 9 V, X. Maturity periods for both sexes are incompletely known.

ETYMOLOGY. Specific epithet mallorcensis, of Majorca, where the species was found.

\section{Comments}

As noted by Lehtinen [1967] there is considerable intraspecific variation in Lathys. Thus, care should be taken in separating the rather similar species of the $L$. stigmatisata-group, in particular females. Available data indicate that the female of L. mallorcensis sp.n. is smaller than all congroupers, L. pygmaea Wunderlich, 2011 excepted, but size may is not be a reliable character. In most females, perhaps all, there is a pair of circular epigynal openings separated by a factor of their diameter ranging from 0.1 (nearly touching) to 1.8 (widely separated). The degree of separation is a helpful character in distinguishing the species, but many species shows overlap. Separation of males also poses some problems. The shape of the screwed terminal part of the conductor is similar in some species. However, the conductor of L. mallorcensis sp.n. is distinctive having a long and straight section at the very tip that is 


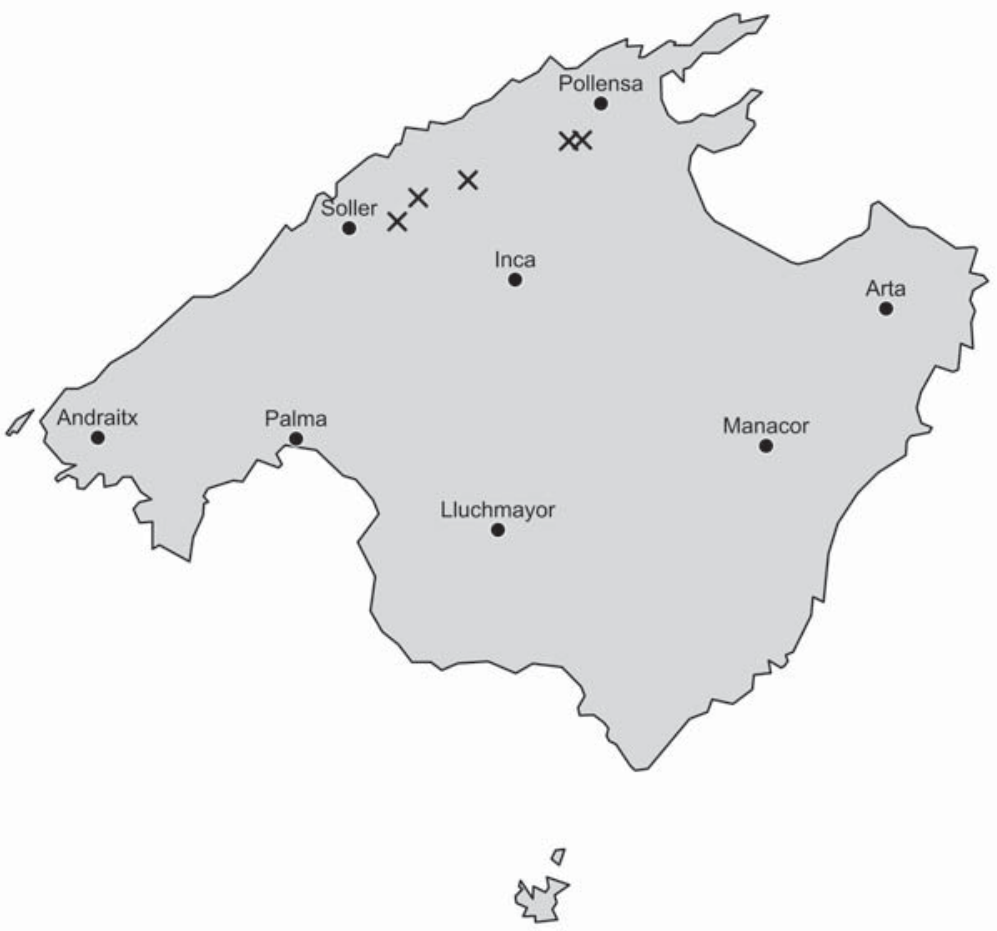

Fig. 12. Lathys mallorcensis sp.n., distribution in Majorca is marked with crosses.

Рис. 12. Lathys mallorcensis sp.n., карта распространения.

parallel with palpal tibia in retrolateral view $(\mathrm{Ctt}$ in Fig. 4), the shape nearly identical in the males inspected. In all other species for which illustrations exist of the palp in retrolateral view this tip is either short and curved or is oriented perpendicular to palpal tibia. It is possible that the position of base of embolus and morphology of seminal ducts on inspecting detached bulb in dorsal view are the best characters to separate males. Illustrations are available for L. mallorcensis sp.n, $L$. alberta Gertsch, 1946, L. stigmatisata and L. spasskyi Andreeva et Tyschchenko, 1969 and in each of these four species these structures are distinctive (compare figs in Marusik et al. [2006 (Fig. 21); 2015 (Figs. 51, 53)] with fig. 9). Lathys mallorcensis sp.n. shows some variation in cheliceral dentition and this character thus seems of limited value in separating the species. Conspecificity with two badly described species from Europe, L. lutulenta Simon, 1914 and L. lepida O. Pickard-Cambridge, 1909 of unknown group affiliation, is not considered an option as these species possess clearly annulated legs according to the original descriptions. As L. mallorcensis sp.n. is with a pair of epigynal openings it is easily separated from other Lathys species that may occur in Majorca (L. narbonensis, $L$. humilis, L. heterophthalma) since these species have a single round or transverse epigynal opening and/or is with clearly annulated legs. The male palps of these species are also distinctly different. There is only limited literature on the occurrence of Lathys in the Balearic Islands. The studies of Orghidan et al. [1975] and
Pons [2004] list records restricted to Majorca and the Cabrera archipelago. In the study of Orghidan, Lathys females found under a stone at Illetas and at Foret de Ca'n Sion, Majorca were assigned to "Scotolathys cf. heterophthalma" (=Lathys heterophthalma Kulczyńs$\mathrm{ki}, 1891)$. Occurrence in Majorca is possible as the species has been recorded near Toulouse in southern France [Déjean, 2015]. The study of Orghidan also lists records of female Lathys narbonensis (Simon, 1876) from Illetes near Calvià and from Bosc de Ca'n Sion near Pollença. The original description of this species [Simon, 1914] was inadequate and lacks detailed figures which make identification of L. narbonensis difficult. Later studies attempting to assign specimens to this species may not all have been correct. The illustrations of epigynes in Denis [1947] and Ledoux et al. [2008] do not seem to agree, also the illustration of the male palp in Noflatscher [1993] may relate to a different species. Noflatscher did not identify his male, merely only proposed a match with $L$. narbonensis. Thus, listing at the World Spider Catalog should perhaps await comparison of Noflatschers male with type material in order to confirm conspecificity. As in Noflatschers study, specimens collected at St. Elm in the present study could not be assigned to L. narbonensis with certainty. It is hoped to deal with this problem in a later paper. Pons [2004] lists several records of juvenile specimens presumably belonging to Lathys humilis (Blackwall, 1855) from various islands in the Cabrera archipelago where it is stated as commonly 
found on bushes along the coast. Recently, one adult female was recorded from Es Tudons, Menorca, confirming the occurrence of this species in the Balearic Islands [Barrientos, Febrer, 2017].

Acknowledgements. Mykola Kovblyuk and Yuri Marusik are thanked for many good comments on the manuscript.

\section{References}

Almquist S. 2006. Swedish Araneae, part 2 - families Dictynidae to Salticidae // Insect Systematics \& Evolution, Supplement. Vol.63. P.285-601.

Andreeva E.M. 1976. [Spiders of Tajikistan]. Dushanbe. 196 p. [In Russian]

Barrientos J.A., Febrer J.B. 2017. Arañas (Arachnida, Araneae) de Menorca (Islas Baleares, España). Nuevos datos // Revista ibérica de Aracnología. T.31. P.8-24.

Bosmans R., Baert L., Bosselaers J., Koninck H., De, Maelfait J.P., Keer J., Van 2009. Spiders of Lesbos (Greece). A catalogue with all currently known spider reports from the Eastern Aegean Island of Lesbos // Newsletter of the Belgian arachnological Society. Vol.24. P.1-72.

Caporiacco L., di. 1934. Aracnidi dell'Himalaia e del Karakoram, raccolti dalla Missione italiana al Karakoram (1929-VII) Memorie della Società Entomologica Italiana. Vol.13. P.113160 .

Chamberlin R.V., Gertsch W.J. 1958. The spider family Dictynidae in America north of Mexico // Bulletin of the American Museum of Natural History. Vol.116. P.1-152.

Déjean S. 2015. Lathys nielseni (Schenkel, 1932) (Araneae, Dictynidae), une espèce confirmée pour la faune de France découverte sur la commune de Toulouse // Revue Arachnologique. Série 2. T.2. P.20-22.

Danilov S.N. 1994. [Cribellate spiders (Aranei, Cribellatae) of Transbaicalia] // Entomologicheskoe Obozrenie Vol.73. P.200209 [in Russian, with English summary].

Denis J. 1937. On a Collection of Spiders from Algeria // Proceedings of the Zoological Society of London. Vol.1936. P.10271060 .

Denis J. 1947. Araignées de France. I. Araignées de Vendée avec la description d'une espèce nouvelle des Pyrénées-Orientales // Revue Française d'Entomologie. T.14. P.145-155.

Kaston B.J. 1978. How to know the spiders. 3rd ed. Dubuque, Iowa: Wm. C. Brown Company Publishers. 272 p.

Koçyi it H.O., Demir H., Seyyar O. 2016. The spider fauna of Hasan Mountain in Turkey // Serket. Vol.15. P.8-29.

Kovblyuk M.M., Kastrygina Z.A., Omelko M.M. 2014. New Lathys Simon, 1884 species from Crimea (Aranei: Dictynidae) // Arthropoda Selecta. Vol.23. No.2. P.195-198.

Ledoux J., Braud S., Duffey E., Chéreau L. 2008. De araneis Galliae II, 9 Lathys sexpustulata Simon $1878=$ Lathys alticola // Revue arachnologique. T.17. P.59-61.

Lehtinen P. 1967. Classification of the cribellate spiders and some allied families, with notes on the evolution of the suborder
Araneomorpha // Annales Zoologici Fennici. Vol.4. No.3. P.199-468.

Marusik Yu.M., Esyunin S.L., Tuneva T.K. 2015. A survey of Palaearctic Dictynidae (Araneae). 1. Taxonomic notes on Dictynomorpha Spassky, 1939, Brigittea Lehtinen, 1967 and Lathys Simon, 1884 // Zootaxa. Vol.3925. No.1. P.129-144.

Marusik Yu.M., Koponen S., Fritzén N.R. 2009a. On two sibling Lathys species (Araneae, Dictynidae) from northern Europe // Stoev P., Dunlop J., Lazarov S. (eds.). A life caught in a spider's web. Papers in arachnology in honour of Christo Deltshev. ZooKeys. Vol.16. P.181-195.

Marusik Yu.M., Kovblyuk M.M., Nadolny A.A. 2009b. A survey of Lathys Simon, 1884, from Crimea with resurrection of Scotolathys Simon, 1884 (Aranei: Dictynidae) // Arthropoda Selecta. Vol.18. No.1-2. P.21-33.

Marusik Yu.M., Ovchinnikov S.V., Koponen S. 2006. Uncommon conformation of the male palp in common Holarctic spiders belonging to the Lathys stigmatisata group ( Araneae, Dictynidae) // Bulletin of the British Arachnological Society. Vol.13. Pt.9. P.353-360.

Nentwig W., Blick T., Gloor D., Hänggi A., Kropf C. 2017. Spiders of Europe. Version 03.2017. Available from: www.araneae. unibe.ch (accessed on September 7, 2017).

Noflatscher M. 1993. Beiträge zur Spinnenfauna Südtirols — IV: Epigäische Spinnen am Vinschgauer Sonnenberg // Berichte des naturwissenschaftlichen-medizinischen Verein Innsbruck. Bd.80. S.273-294.

Ono H., Ogata K. 2009. Titanoecidae, Dictynidae // Ono H. (ed.). The Spiders of Japan with keys to the families and genera and illustrations of the species. Kanagawa: Tokai University Press. P.132-139.

Orghidan T., Dumitresco M., Georgesco M. 1975. Mission biospéologique "Constantin Dragan" à Majorque (1970-1971). Première note: Arachnides (Araneae et Pseudoscorpionidea) // Travaux de 1'Institute de Spéologie "Émile Racovitza". T.14. P.9-33.

Pons G. 2004. Biogeografia, ecologia i taxonomia de les aranyes (arachnida, araneae) de les illes balears. Models de distribució de la fauna insular. Universitat de les Illes Balears. 542 p.

Simon E. 1911. Catalogue raisonné des arachnides du nord de l'Afrique (1re partie) // Annales de la Société entomologique de France. T.79. P.265-332.

Simon E. 1914. Les Arachnides de France. T.6. Le Synopsis général et le catalogue des espèces françaises de l'ordre des Araneae; 1re partie. Paris. 308 p.

World Spider Catalog. 2017. World Spider Catalog. Natural History Museum Bern, version 18.5. Available from: http:// wsc.nmbe.ch. (December 14, 2017).

Wunderlich, J. 1992. Die Spinnen-Fauna der Makaronesischen Inseln: Taxonomie, Ökologie, Biogeographie und Evolution // Beiträge zur Araneologie Bd.1. S.1-619.

Wunderlich, J. 2011. Extant and fossil spiders (Araneae) // Beiträge zur Araneologie Vol.6. P.1-640.

Zhang Z., Hu D., Zhang Y. 2012. Notes on the spider genus Lathys Simon, 1884 (Araneae: Dictynidae), with description of four new species from China // Zootaxa. Vol.3359. P.1-16.

Responsible editors Yu.M. Marusik, K.G. Mikhailov 